\title{
Optimal and Collaborative Rate Selection for Interference Cancellation in Wireless Networks
}

\author{
Vangelis Angelakis, Lei Chen and Di Yuan
}

Linköping University Post Print

N.B.: When citing this work, cite the original article.

(C)2011 IEEE. Personal use of this material is permitted. However, permission to reprint/republish this material for advertising or promotional purposes or for creating new collective works for resale or redistribution to servers or lists, or to reuse any copyrighted component of this work in other works must be obtained from the IEEE.

Vangelis Angelakis, Lei Chen and Di Yuan, Optimal and Collaborative Rate Selection for Interference Cancellation in Wireless Networks, 2011, IEEE Communications Letters, (15), 8, 819-821.

http://dx.doi.org/10.1109/LCOMM.2011.062711.110561

Postprint available at: Linköping University Electronic Press

http://urn.kb.se/resolve?urn=urn:nbn:se:liu:diva-70529 


\title{
Optimal and Collaborative Rate Selection for Interference Cancellation in Wireless Networks
}

\author{
Vangelis Angelakis, Lei Chen, and Di Yuan \\ Department of Science and Technology, Linköping University, SE-601 74 Norrköping, Sweden. \\ E-mails: \{vanan, leich, diyua\}@itn.liu.se
}

\begin{abstract}
Analysis of wireless systems commonly assumes single-user detection at the receivers. Interference is typically treated as noise. On the other hand, multiuser detection has long been taking advantage of interference cancellation (IC) to increase capacity. We exploit IC by optimal rate selection. Transmission rates are collaboratively optimized to maximize the benefit of IC. A link reduces its rate, if that enables IC to significantly boost the SINR on other links. We provide a complexity analysis and an integer programming model to find the optimal IC pattern. Simulation results indicate that throughput improvement is over $30 \%$ in low SINR regimes.
\end{abstract}

Index Terms - Interference cancellation; rate selection; optimization; wireless communications.

\section{INTRODUCTION}

In wireless networking, the typical receiver model assumed is that of single-user detection. As a result, interference from concurrent transmissions is treated as noise and regarded as a performance-limiting factor. Multiuser detection [10] enables receivers to cancel interference provided that it is received with sufficient strength. Various multiuser detection techniques have been developed, such as successive interference cancellation [1], [8], parallel interference cancellation [3] and more recently zero forcing and interference alignment [5], [7]. Rate adaptation for an IC-enabled cognitive radio system was studied in [9] to derive achievable rate regions. Recently, SINR balancing with IC was investigated in [6].

The novelty in our work is the introduction of a scheme that actively optimizes the transmission rates, to maximize the benefit of IC in terms of the overall system throughput. In wireless networking, a link will typically operate at the highest rate permitted by the SINR. The key observation is that, with IC, this may not be optimal. Some links may, by reducing their transmission rate, enable others to perform IC on them to get better SINR and increased rate. An illustrative example is given in Figure 1. If both links operate at the highest rates (indicated by the gray bars) allowed by their respective SINRs, no IC can take place. If link 1 uses a lower rate (the left black bar) it enables link 2 to perform IC and reach a higher SINR, supporting a significantly higher rate (the right black bar).

Implementing IC is not technically trivial, primarily due to synchronization issues. To facilitate the flexibility of collaboratively identifying the optimal rates, the links must be able to exchange information regarding SINR conditions and rate decisions. Therefore the issue of the coordination overhead arises in the context of a distributed implementation of the proposed scheme. Also, QoS constraints may prohibit some links from

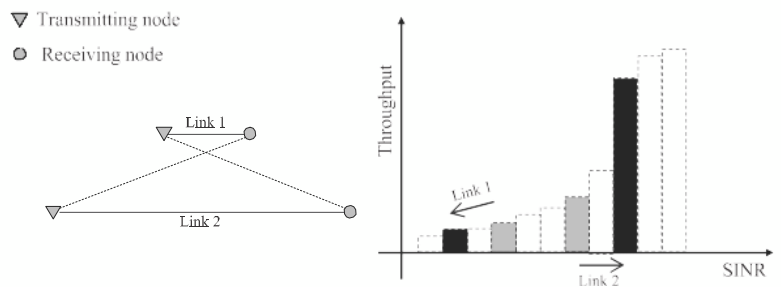

Fig. 1. A two-link example of collaborative rate selection with IC.

reducing their rate. Thus the optimal IC solution might not be feasible in all contexts. Our study, therefore provides the upper bound on the expected gains when implementing collaborative rate selection with IC in a wireless network.

The contributions of this letter are: 1) the problem formulation of throughput maximization by collaborative rate selection with single link and parallel IC, and the theoretical result on its complexity, 2) the proposal of an integer optimization model for performance benchmarking, and 3) the insight into the benefits of rate selection with IC.

\section{System Model AND PRoblem Complexity}

We assume a nondecreasing and stepwise rate function, mapping SINR values to throughput. Each available modulation and coding scheme corresponds to a rate level, with a respective throughput value. Let $L$ be the number of levels. Consider a wireless network where $K$ links have been admitted for concurrent transmission. That is, without any IC, all links have sufficient SINR to achieve some non-zero rate level in the rate function. Transmission power is fixed, but may be varied by transmitter.

We do not restrict our discussion to a particular propagation model, nor impose any further assumption on the rate function. We formulate the generic problem setting as follows. We denote by $C^{k}$ the set of all the subsets of links that link $k$ can cancel, and by $l_{k}^{k \backslash H}$ the rate level attained by link $k$ when it cancels all links in set $H, H \in C^{k}$. Similarly, we denote by $l_{h}^{k \backslash h}$ the highest rate level that link nhay operate on, in order to be cancelled by link $k$. We denote by $l_{k}^{k}$ the (initial) rate level of $k$ without any IC. For each of these rate levels the attained throughput is respectively: $r^{l_{k}^{k \backslash H}}, r_{h}^{l_{h}^{k \backslash h}}$, and $r^{l_{k}^{k}}$.

The problem is to use rate selection to find the optimal IC pattern, maximizing the total throughput, as formalized below. Input: A set of links, and for each link $k$ and for each set $H \in C^{k}$, the values of: $l_{k}^{k}, l_{k}^{k \backslash H}, l_{h}^{k \backslash h}, r^{l_{k}^{k}}, r^{l_{k}^{k \backslash H}}$, and $r^{l_{h}^{k \backslash h}} \forall h \in H$. 
TABLE I

THROUGHPUT VALUES USED IN THE COMPLEXITY PROOF

\begin{tabular}{lc|lc}
$\begin{array}{c}\text { Link pair } \\
\text { Condition }\end{array}$ & $\begin{array}{c}x_{1}, \widehat{x}_{1} \\
\text { Throughput }\end{array}$ & \multicolumn{2}{c}{$\begin{array}{c}\text { Cink generated by clause } k \\
\text { Throughput }\end{array}$} \\
\hline \hline$l_{x_{1} \backslash \widehat{x}_{1}, l_{\widehat{x}_{1} \backslash x_{1}}^{x_{1}}}^{x_{1}}$ & $n^{2}$ & $l_{k}^{k \backslash \widehat{x}_{1}}, l_{k}^{k \backslash x_{2}}, l_{k}^{k \backslash x_{3}}$ & $n$ \\
$l_{x_{1} \backslash x_{1}}^{x_{1}}, l_{\widehat{x}_{1} \backslash \widehat{x}_{1}}^{x_{1}}$ & 1 & $l_{\widehat{x}_{1} \backslash \widehat{x}_{1}}^{k}, l_{x_{2}}^{k \backslash x_{2}}, l_{x_{3}}^{k \backslash x_{3}}$ & 1 \\
$l_{x_{1}}^{x_{1}}, l_{\widehat{x}_{1}}^{\widehat{x}_{1}}$ & 1 & $l_{k}^{k}$ & 1 \\
\hline
\end{tabular}

Output: The IC pattern in the form of $d \bar{n} \times K$ binary matrix, which maximizes the aggregate throughput. The matrix elements $b_{i j}, i=1, \ldots, K, j=1, \ldots, K$ are defined as follows.

- If link $k$ cancels the interference of link $h$, then $b_{k k}=0$, $b_{k h}=1$. Note that if link $k$ performs IC on more than one link concurrently, then there will be multiple ones in the row. - If link $k$ performs no IC at all, thep $=1$ and the remaining elements of the row are all zeros.

We prove the NP-hardness of the rate selection problem by a reduction from the well-known 3-satisfiability (3-SAT) problem [4]. The 3-SAT problem is a decision problem determining if there are values that can be assigned to then variables in a given boolean formula to make it true. The formula consists of the conjunction of $n$ clauses. Each clause is the disjunction of exactly 3 literals taken from the set of tha variables. A literal may be either the variable $x_{i}$ for $=1, \ldots, m$ or its negation $\widehat{x}_{i}$.

$n$

use it to construct an instance of our system, where each of the diterals is a link. For the proof, each link generates (receives) interference only to (from) the links representing the negation literal in the 3-SAT instance. Furthermore, the

we construct more links - one per clause. Each such link receives interference by the links corresponding to the literals contained in the clause. Figure 2 displays part of the construction, where the solid arrows indicate the links while the dotted ones indicate potential interference. Note that the three vertical pairs of Figure 2 will connect to as many horizontal links as the number clauses that contain any of the six literals. The link throughput values are set in Table I.

Lemma 1: There is an optimal solution where for each link $x_{i}, i=1 \ldots m$ either $b_{x_{i} \widehat{x}_{i}}=1$, or $b_{\widehat{x}_{i} x_{i}}=1$.

Proof: Arbitrarily setting $\widehat{\widehat{x}}_{i} \boldsymbol{x}_{i}$ or $b_{\mathrm{x}_{i}} \mathrm{tg}_{i} 1$, gives a total throughput value of at least $m n^{2}+m+n^{2}$. If $b_{\widehat{x}_{i} x_{i}}=b_{x_{i} \widehat{x}_{i}}=0$ for some $i$ then the total throughput would be at most $(m-$ 1) $n^{2}+(m-1)+2+n n=m n^{2}+m+1$.

Lemma 2: For the clause link $k$ shown in Figure 2, the

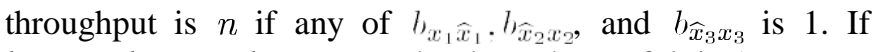
$b_{\widehat{x}_{1} x_{1}}=b_{x_{2} \widehat{x}_{2}}=b_{x_{3} \widehat{x}_{3}}=1$, the throughput of $k$ is 1 .

Proof: If the condition in the first part of the lemma holds, then at least one of $\widehat{x}_{1}, x_{2}, x_{3}$ has throughput 1 (Table I), so link $k$ can perform IC on it, thus reaching throughput $n$. Otherwise, $k$ cannot perform any IC and so its rate is 1 .

Theorem 1: The decision problem of rate selection with IC is NP-complete.

Proof: From the lemmas above, it follows that the original 3-SAT formula will be true if the optimal throughput is

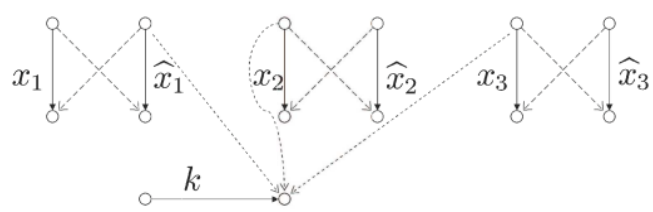

Fig. 2. The links constructed from clause $\left(\widehat{x}_{1} \vee x_{2} \vee x_{3}\right)$. Solid arrows indicate links in the system, while dotted ones indicate significant interference.

exactly: $m n^{2}+m+n$, and false if the optimal throughput is at most $m n^{2}+m+n(n-1)+1$ Therefore the optimal throughput gives the answer to the 3-SAT problem. Hence the conclusion.

Although the problem is challenging in its theoretical computational complexity, we develop an integer programming model, presented in the next section, to find the global optimal solution fast for reasonably large numbers of links.

\section{The Integer Programming Model}

We introduce two sets of binary variables: $x_{k}^{l}$, which is 1 when $\operatorname{link} k$ is using rate level $l$, otherwise 0 , any $k_{k H}$, which is 1 if link $k$ cancels the interference of all links in $H$, otherwise 0 . The integer programming model to maximize the total system throughput is:

$$
\begin{gathered}
\text { maximize: } \sum_{k=1}^{K} \sum_{l=1}^{L} r^{l} x_{k}^{l} \\
\text { s.t.: } \sum_{l=1}^{L} x_{k}^{l}=1, \quad k=1 \ldots K \\
\sum_{l: l \leq l_{k}^{k \backslash H}} x_{k}^{l} \geq y_{k \backslash H}, k=1 \ldots K, \forall H \in C^{k} \\
\sum_{l: l \leq l_{h}^{k \backslash h}} x_{h}^{l} \geq y_{k \backslash H} \cdot k=1 \ldots K, \forall H \in C^{k}, \forall h \in H \\
\sum_{l: l \leq l_{k}^{k}} x_{k}^{l} \geq 1-\sum_{H \in C^{k}} y_{k \backslash H}, k=1 \ldots K
\end{gathered}
$$

The sum in (1) is the total throughput of all the links. Equation (2) is used to guarantee that each link uses exactly one rate level, thus keeping all links active. Constraint (3) captures that if link $k$ cancels the interference from the links of $H$, the level attained by $k$ can not go above that of $l_{k}^{k \backslash H}$. The construction of (4) guarantees that if a link is cancelled byk, then $h$ 's throughput can not go above level $l_{h}^{k \backslash h}$. Finally, (5) states that if for link kno IC takes place at all, then the throughput achieved by $k$ will not go above the original level $l_{k}^{k}$

\section{Simulation Results}

To numerically evaluate the potential of rate selection with IC, we use instances of randomly placed nodes in a circular area of $1 \mathrm{~km}$ radius. The path loss parameter follows the free space model. We assume that all transmitting nodes use a power of 1 Watt, the noise power spectral density is $174 \mathrm{dBm} / \mathrm{Hz}$ and the nodes operate at $2 \mathrm{GHz}$ using a 5 $\mathrm{MHz}$ channel. The throughput function $R$ maps SINR to nine throughput values, using the modulation and coding schemes shown in Table II. The lowest and highest throughput 
TABLE II

THROUGHPUT FUNCTION PARAMETERS

\begin{tabular}{ll}
\hline \hline Channel Bandwidth: & $5 \mathrm{MHz}$ \\
Oversampling: & $144 / 125$ \\
Modulation schemes: & BPSK, QPSK, 16QAM. 64QAM \\
Coding Rates: & $1 / 4,1 / 2,2 / 3,3 / 4$ \\
Bit Error Rate: & $10^{-4}$ \\
\hline
\end{tabular}

values are $800 \mathrm{Kbps}$ and $12.8 \mathrm{Mbps}$, corresponding to SINR thresholds of $5.3 \mathrm{~dB}$ and $23.6 \mathrm{~dB}$ respectively.

We have conducted three sets of experiments with SINR threshold $\gamma$ being equal to $-2 \mathrm{~dB}, 0 \mathrm{~dB}$, and $2 \mathrm{~dB}$. For each of them, transmitter-receiver pairs meeting the threshold are selected among the nodes to form links, and the baseline throughput without IC was calculated. The number of links spanned between two and nine; for the parameter setting under consideration, this is consistent with the size of feasible link activation sets [2]. Enabling IC rate optimization may bring down the rates of some links, but note that it is not allowed to remove a link from the system in order to benefit others.

We split our simulations into two groups: (i) single link IC, allowing cancellation of up to one interferer per link, and (ii) parallel IC, allowing a link to perform multiple cancellations concurrently. Each setup is run for 50 node placement instances and the results presented are the mean values.

Table III shows the results in terms of the number of cancellations. Note that the number of cancellations that take place is practically the same regardless of allowing up to one, or multiple parallel cancellations. Indeed, the single-link and parallel IC patterns differ only for the $-2 \mathrm{~dB}$ SINR threshold. This implicitly verifies one of the key findings in [11], namely that removing the impact of one of the interfering nodes yields the most of the performance gain from IC.

Figure 3 shows the amount of improvement in the overall throughput for single-link IC. It is clear that the IC gain in throughput is much higher in the low SINR regime: For two links, rate selection with IC practically doubles the throughput, and for up to nine links, the gain remains above $30 \%$. Note that as the number of links grows, more links can find an interferer to cancel, still, the gain is less due to denser topology. When more nodes are randomly packed in the same area, there is a better chance for a transmitter to be close enough to a receiver to allow IC. On the other hand, the overall interference is inherently high in the system, so the likelihood of significant rate improvement via multiple cancellations is smaller, since each of the cancellations has to face several strong interferers.

\section{CONCLUSION}

We have considered a novel problem setting of collaboratively optimizing the transmission rates to explore the potential of interference cancellation in wireless networking. Results ranging from problem complexity to numerical evaluation have been presented. The integer programming formulation can act as a platform for performance benchmarking. The study shows that the performance gain of collaborative rate optimization with interference cancellation is quite significant for the low SINR regime. Motivated by these numerical results,
TABLE III

AVERAGE NUMBER OF CANCELLED LINKS

\begin{tabular}{|c|c|c|c|c|c|c|}
\hline & \multicolumn{3}{|c|}{ Single IC } & \multicolumn{3}{|c|}{ Parallel IC } \\
\hline$\gamma($ in $\mathrm{dB})$ : & -2 & 0 & 2 & -2 & 0 & 2 \\
\hline \# of Links & & & & & & \\
\hline 2 & 1.0 & 0.8 & 0.6 & 1.0 & 0.8 & 0.6 \\
\hline 3 & 1.0 & 1.2 & 0.4 & 1.0 & 1.2 & 0.4 \\
\hline 4 & 1.0 & 1.2 & 1.2 & 1.0 & 1.2 & 1.2 \\
\hline 5 & 2.0 & 0.4 & 1.6 & 2.0 & 0.4 & 1.6 \\
\hline 6 & 2.2 & 0.2 & 1.0 & 2.2 & 0.2 & 1.0 \\
\hline 7 & 2.2 & 0.4 & 0.2 & 2.0 & 0.4 & 0.2 \\
\hline 8 & 3.0 & 0.6 & 0 & 2.8 & 0.6 & 0 \\
\hline 9 & 3.6 & 0 & 0.2 & 3.6 & 0 & 0.2 \\
\hline
\end{tabular}

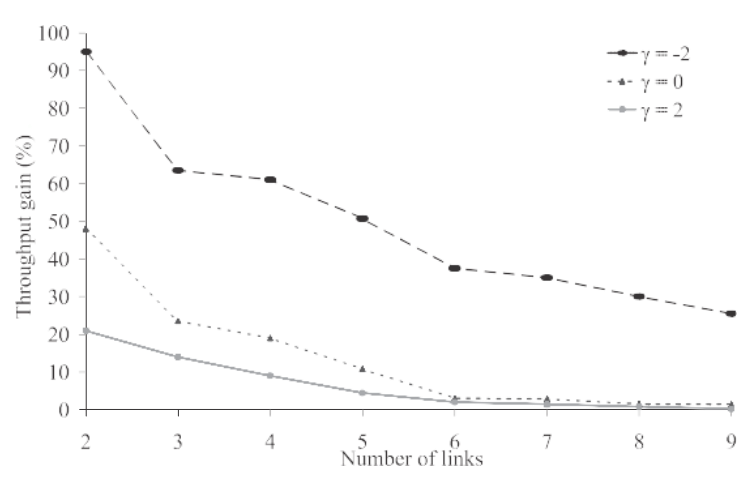

Fig. 3. Total throughput gain vs. number of links for various thresholds.

it is of interest to perform additional analysis of the gain in the low SINR regime, since in this case mutual interference cancellations are more likely to take place. Such an analysis can open up a new outlook on link admission strategies that explore small clusters of low SINR links that with interference cancellation can significantly boost the system throughput.

-This work has been supported by: the Swedish excellence center ELLIIT, CENIIT, Linköping University, and the EU FP7-People-2007-3-1-IAPP-218309 Marie Curie project.

\section{REFERENCES}

[1] A. Agrawal, et al. "Power control for successive interference cancellation with imperfect cancellation," in Proc. IEEE ICC '02, pp. 356-360, Apr. 2002 ,

[2] S. Borbash and A. Ephremides, "The feasibility of matchings in a wireless network," IEEE/ACM Trans. Networking, vol. 14, pp. 2749-2755, Jun. 2006.

[3] D. R. Brown III, et al. "On the performance of linear parallel interference cancellation," in IEEE Trans. Inf. Theory, vol. 47, pp. 1957-1970, July 2001.

[4] M. R. Garey and D. S. Johnson, Computers and Intractability: A Guide to the Theory of NP-Completeness, W. H. Freeman, 1979.

[5] D. Halperin, T. Anderson, and D. Wetherall, "Taking the sting out of carrier sense: interference cancellation for wireless LANs," in Proc. ACM MobiCom '08, pp. 339-350, Sep. 2008.

[6] E. Karipidis, E. G. Larsson, and D . Yuan, "Mixed-integer linear programming framework for max-min power control with single-stage interference cancellation," in Proc. IEEE ICASSP '11, pp. 3448-3451, May 2011.

[7] L. E. Li, et al. "A general algorithm for interference alignment and cancellation in wireless networks," in Proc. IEEE INFOCOM '10, pp. 1-9, Mar. 2010.

[8] P. Patel and J. Holtzman, "Analysis of a simple successive interference cancellation scheme in a DS/CDMA system," IEEE J. Select. Areas Commun., vol. 12, pp. 796-807, June 1994.

[9] P. Popovski, et al. "Opportunistic interference cancellation in cognitive radio systems," in Proc. IEEE DySPAN, pp. 472-475, Apr. 2007.

[10] S. Verdu, Multiuser Detection, Cambridge University Press, 1998.

[11] S. Weber, et al. "Transmission capacity of ad hoc networks with successive interference cancellation," IEEE Trans. Info. Theory, vol. 53, pp. 2799-2814, Aug 2007. 